\title{
Preventing pneumococcal disease: A Canadian consensus conference, February 16 to 18,1998
}

$\mathrm{S}$ treptococcus pneumoniae is an important cause of illness, hospitalization and death worldwide. It is responsible for meningitis, pneumonia, otitis media, sinusitis and bacteremia in adults and children. Among all adult community-acquired pneumonias requiring hospital admission, $S$ pneumoniae ranks first among known microbial causes and accounts for approximately $30 \%$ to $50 \%$ of such cases (1). It is estimated that in nonindustrialized countries pneumococcal pneumonia results in the death of over one million children each year, half of whom are younger than one year of age (2). In industrialized countries, invasive disease due to $S$ pneumoniae is a serious problem among the elderly, infants and those with chronic underlying medical conditions or immune systems that are compromised either because of disease or immunosuppressive drug therapy.

In the United States, pneumococcal disease is estimated to account for 3000 cases of meningitis, 50,000 cases of bacteremia, 500,000 cases of pneumonia and 7,000,000 cases of otitis media each year (3). The incidence of invasive pneumococcal disease has not been well defined in Canada until relatively recently. Population-based surveillance for invasive pneumococcal disease in Metropolitan Toronto/Peel region (population 3.4 million) revealed an overall incidence of 14.4 cases per 100,000 population in $1995,16.1$ cases per 100,000 population in 1996 and 11.8 cases per 100,000 population in 1997 (4). Ninety-two per cent of cases were caused by serotypes contained in the current 23 -valent pneumococcal vaccine, and $76 \%$ of cases belonged to one or more of the high risk groups defined by the National Advisory Committee on Immunization (NACl) (4). The Sentinel Health Unit Surveillance System (SHUSS), an active population-based surveillance for laboratory-confirmed disease conducted in nine health units within eight Canadian provinces in 1996, revealed an overall incidence of 15.1 cases of invasive pneumococcal disease per 100,000 population (5). The age-specific incidence was greatest in children under five years of age $(55.3$ cases per 100,000) and in persons 65 years of age or over (46.4 cases per
$100,000)$. Ninety-four per cent of cases were caused by serotypes contained in the 23-valent pneumococcal vaccine (5).

Historically, $S$ pneumoniae was uniformly susceptible to penicillin. The first isolate resistant to penicillin was reported from Australia in 1967 (6). The incidence of penicillin resistance in many areas of the world has been steadily increasing during the past two decades but has grown at a much higher rate in the past five years (7). The National Centre for Streptococcus (NCS), Edmonton, Alberta, a voluntary passive reporting system, found that $7.8 \%$ of isolates submitted between 1992 and 1995 had diminished susceptibility to penicillin (8). During $1996 / 97$, this proportion had increased to $10.2 \%$ (9). SHUSS identified $7.4 \%$ of isolates having diminished susceptibility to penicillin in 1996 (5). Vancomycin is one of the few antimicrobials to which reduced susceptibility has not been shown. The increase in antibiotic-resistant $S$ pneumoniae underscores the need for prevention.

A 23-valent polysaccharide pneumococcal vaccine has been available since the 1980 s. NACI recommends vaccination for specified groups at high risk of pneumococcal disease; however, vaccine programs and policies for who receives publically funded vaccines are the responsibility of each of the provinces and territories (10). Only recently have public health programs in some provinces and territories begun to promote the vaccine's use.

The need for national consensus regarding the control of pneumococcal disease was recognized. To identify initiatives currently under way in Canada, including publicly funded immunization programs, surveillance, epidemiological research and laboratory activities, and to identify priority areas for the future, the Laboratory Centre for Disease Control (LCDC), Health Canada, Ottawa, Ontario, organized a meeting of national and international stakeholders. The meeting, entitled "Preventing Pneumococcal Disease: A Canadian Consensus Conference" was held in Ottawa, Ontario, February 16 to 18 , 1998. The objectives were to achieve national consensus on the following issues: 
- surveillance of pneumococcal disease;

- immunization and publicly funded campaigns;

- epidemiological research;

- antimicrobial-resistant $S$ pneumoniae; and

- laboratory activities as they relate to $S$ pneumoniae.

The emphasis of the conference was to generate national recommendations on the use of pneumococcal vaccine. The conference began with a series of presentations by national and international authorities in the area of pneumococcal disease. Participants were then divided into working groups. Each group was asked to consider specific questions when developing their recommendations. The proposed recommendations from each group were then presented to and discussed by the plenary. A consensus process was used to arrive at the final recommendations, in which consensus was broadly defined as that which a majority of participants strongly supported, with acceptance by the rest.

This report presents the final consensus recommendations from each working group. Recommendations from the Consensus Conference are broken down into four categories: Surveillance and Targeted Research; Immunization - Role in Disease Prevention; Immunization Programs - Delivery and Promotion Strategies; and Program Evaluation and Monitoring.

\section{RECOMMENDATIONS}

\section{Surveillance and targeted research}

Invasive pneumococcal disease is not a reportable condition in every province or territory of Canada. However, recent Canadian studies and surveillance activities have better quantified the burden of disease attributable to invasive pneumococcal infections and have identified increasing trends in resistance to penicillin $(4,5,8,9)$. While there is some Canadian information regarding penicillin resistance, systematic collection of this data in many areas of Canada is not occurring because drug-resistant $S$ pneumoniae is not a reportable condition. This information is needed to provide clinicians with data on local drug resistance patterns to guide empirical therapy of serious pneumococcal infections.

The incidence of drug-resistant infections can change rapidly. In some areas of the United States, as many as 30\% of pneumococcal isolates are resistant to penicillin $(11,12)$. Laboratory-based surveillance for invasive pneumococcal disease is critical for monitoring both antibiotic resistance and the serotypes causing disease. With increased use of the 23valent polysaccharide vaccine, it will also be important to monitor $S$ pneumoniae incidence and the groups at risk of disease, and to observe changes over time.

Recommendation 1: A national system of surveillance should be established for invasive pneumococcal disease. The surveillance system should include monitoring of serotypes and antimicrobial resistance. All pneumococcal surveillance systems should periodically compare and share information to eliminate gaps in surveillance and obtain the widest possible perspective on invasive pneumococcal disease.

Recommendation 2: Invasive pneumococcal disease should be made nationally notifiable and reportable within all provinces and territories, with minimal core data for all reported cases. Isolates from blood, and cerebrospinal, articular, peritoneal or pleural fluid should be included but not those isolates from sputum or bronchoalveolar fluid. Common definitions should be used for disease reporting.

Recommendation 3: The collection of a representative sample of isolates in each province, territory or region should be coordinated by a steering committee of epidemiologists and microbiologists, who would receive monthly information from all laboratories in the province on the number of invasive $S$ pneumoniae cases detected from normally sterile sites and the number of these cases resistant to penicillin, as determined by the oxacillin screen. In addition, a network of laboratories chosen by the steering committee as representative of the provincial or territorial population should send all isolates from normally sterile sites to the provincial laboratory each month.

Recommendation 4: More detailed information, including risk factor and immunization information, should be collected, and the serotype and antibiotic susceptibility determined for a representative sample of reported cases to monitor the impact of immunization on the epidemiology of pneumococcal disease. These pneumococcal isolates may be sent by the provincial laboratory to the NCS for serotyping and confirmation of antibiotic resistance as required.

Recommendation 5: Information regarding the number of cases of invasive pneumococcal disease and the number with resistance to penicillin and other appropriate antibiotics should be forwarded from provincial public health laboratories to provincial or territorial epidemiologists and the LCDC on a monthly basis

Recommendation 6: Information collected by the surveillance system should be made available at least annually and no later than six months after the end of the year to all those who contribute to its collection and to those who will use it to determine vaccine policy, including NACI and the Advisory Committee on Epidemiology (ACE).

Recommendation 7: SHUSS should periodically monitor invasive pneumococcal isolates to establish disease incidence, populations at risk and the effect of vaccine introduction. These results should be communicated to NACI, LCDC, ACE, Chief Medical Officers of Health ( $\mathrm{CMOH})$ and the Technical Advisory Committee on Public Health Laboratory Directors in a timely manner. SHUSS may also examine vaccine failures to establish etiology.

Recommendation 8: The Canadian Immunization Monitoring Program, Active (IMPACT) system should be considered as an additional surveillance system in preparation for the introduction of conjugate vaccines in children. Until that time, the systems already mentioned should analyze their data for the burden of disease in children.

Recommendation 9: The following issues should be given high priority in the area of research:

- the potential benefits of periodic revaccination with polysaccharide vaccine and the potential for alternative strategies for long term protection, including the use of conjugate vaccines; 
- the efficacy of conjugate vaccines in infants, children and adults;

- vaccine efficacy in certain high risk groups, including people infected with human immunodeficiency virus and those with diabetes;

- the incidence of pneumococcal disease and the prevalence of risk factors for people living in special environments and social settings (including Aboriginal populations);

- monitoring the effectiveness of pneumococcal vaccine after the introduction of immunization programs by linking clinical outcomes, serotypes and vaccination status;

- development of valid indicators of pneumococcal morbidity and mortality; and

- updating the meta-analysis on the effectiveness of immunization delivery strategies.

\section{Immunization - Role in disease prevention}

Studies on the burden of illness attributable to pneumococcal disease in Canada yield results that are similar to studies in other countries $(4,5,13,14)$. For this reason, estimates of pneumococcal vaccine effectiveness and cost effectiveness from other studies may be extrapolated to the Canadian population. In observational studies, the effectiveness of pneumococcal vaccine in preventing invasive pneumococcal disease has generally ranged from $55 \%$ to $80 \%(13,15,16)$. A meta-analysis of nine randomized controlled trials of pneumococcal vaccine concluded that pneumococcal vaccine is efficacious in reducing the frequency of bacteremic pneumococcal pneumonia among adults in low risk groups (17).

Pneumococcal vaccination has been found to be safe and cost effective in preventing invasive disease among elderly people and people with certain chronic medical conditions. In terms of cost per life year gained, it compares favourably with other well accepted preventive and therapeutic interventions, such as influenza immunization, mammography screening and hypertension screening (18). A recent economic analysis of the cost effectiveness of pneumococcal vaccination against bacteremic disease among elderly people in the United States found that pneumococcal vaccination was cost saving in persons 65 years of age and older. Vaccination saved US\$8.27 and gained 1.21 quality adjusted days of life per person vaccinated (19). A cost-utility analysis in Quebec found that pneumococcal immunization compared favourably with other programs and services provided to seniors or persons with chronic conditions (20). Other studies have also indicated that pneumococcal vaccination is cost effective (21).

The demonstration of effectiveness and cost effectiveness are only two of a number of factors involved in the decision to implement a publicly funded immunization program. Other important factors include burden of illness, vaccine safety, antibiotic resistance, political climate, competing health priorities, public demand, media interest and provider interest. The importance of each of these variables in the decision-making process will differ from one province or territory to another.

Recommendations for the use of pneumococcal vaccine are published in the Canadian Immunization Guide (10). However, the incidence of pneumococcal disease in different populations that may be at greater risk of disease because of biological or socioeconomic factors (eg, Aboriginal populations, homeless people, people living in correctional facilities, people with solid tumours) is not always known. The immunization guide is revised by NACI every four years to reflect recent evidence regarding groups at increased risk of disease.

Vaccination may not confer lifelong protection against disease. Strategies that may be considered for the future include revaccination or the use of other vaccines, such as the conjugate vaccines currently under development.

Recommendation 10: The evidence strongly supports the inclusion of pneumococcal vaccine in publicly funded immunization programs in all Canadian provinces and territories. The initial priority of pneumococcal immunization programs should be to offer a single dose of vaccine to all high risk groups as defined by NACI.

Recommendation 11: NACI should develop a statement on adult immunization, with particular reference to high risk groups and Aboriginal peoples. Pneumococcal immunizations should be included in this statement.

Recommendation 12: NACI should communicate with the $\mathrm{Ca}$ nadian Task Force on Periodic Health Examination to develop consistent pneumococcal immunization recommendations.

Recommendation 13: LCDC and vaccine manufacturers should disseminate to each province and territory current data on the benefit of pneumococcal vaccine in preventing disease, rather than attempting to demonstrate, for example, its benefit in preventing pneumonia where $S$ pneumoniae has not been isolated from a normally sterile body fluid.

\section{Immunization programs - Delivery and promotion strategies}

The barriers to pneumococcal immunization in Canada include a lack of awareness by health care workers, the public, and policy makers regarding the burden of the disease, the efficacy and cost effectiveness of pneumococcal immunization, and appropriate adult immunization practices in general; the cost of immunization programs to public health services and, in some cases, the lack of incentive for physicians to provide immunization; and the lack of infrastructure for recording immunization and identifying high risk populations. Immunization registries were the subject of a separate consensus conference meeting in March 1998 (22). Recommendations developed from that meeting are pertinent to pneumococcal immunization.

Studies carried out in the province of Quebec indicate that privately funded immunization programs achieve a low immunization rate (23). As well, evidence on strategies to deliver vaccine indicates that system strategies as opposed to provider or client-oriented strategies are the most effective. Immunization of residents of long term care facilities can be an effective place to begin because it is relatively easy in these facilities to ensure complete vaccine coverage. Promotion and 
implementation of pneumococcal immunization programs can also be facilitated if they are linked with influenza immunization programs that have similar target groups.

Recommendation 14: The following goal and objectives for the control of pneumococcal disease should be adopted throughout the country.

Goal

To reduce illness and death due to pneumococcal disease Objectives

- To achieve a $40 \%$ reduction in the incidence of invasive pneumococcal disease in vaccine-eligible groups by the year 2005

- To achieve a $40 \%$ reduction in the rate of death due to invasive pneumococcal disease in vaccine-eligible groups by the year 2005

Recommendation 15: The following goal and objectives for publicly funded immunization programs should be adopted throughout the country.

Goal

To immunize all persons for whom pneumococcal vaccine is recommended by $\mathrm{NACI}$

Objectives

- To initiate a publicly funded pneumococcal immunization program for all persons for whom vaccine is recommended by NACI by the year 2000 in all provinces and territories

- To encourage provinces and territories to develop an implementation strategy to provide vaccine to all persons for whom the vaccine is recommended by NACI by the year 2000

- To achieve and maintain 95\% vaccine coverage for residents of long term care facilities by the year 2003

- To achieve and maintain $80 \%$ vaccine coverage in all other groups for whom vaccine is recommended by the year 2003

- To review progress towards achievement of vaccine coverage targets in the year 2002

- To ensure that all recipients of influenza vaccine are also appropriately immunized with pneumococcal vaccine, if eligible.

Recommendation 16: Each province and territory should purchase vaccine for all its population at high risk for pneumococcal disease, including Aboriginal peoples and status members of First Nations.

Strategies for delivery of pneumococcal vaccine

Recommendation 17: Concurrent promotion and administration of pneumococcal and influenza vaccine should be done when appropriate. Both vaccination programs should be promoted in the fall, making use of the media (eg, television, radio, press releases, posters and community newspapers).

Recommendation 18: A standard immunization recordkeeping form should be used by all health care providers. Immunization record-keeping systems should be improved at all levels in all provinces and territories to improve access to rec- ords for providers and to encourage the public to keep a record of immunization status.

Recommendation 19: Standing orders and medical directives should be implemented for pneumococcal vaccination of residents on admission to long term care facilities, and for vaccination of persons in high risk groups on discharge from hospitals.

Recommendation 20: Pneumococcal vaccine should be delivered to people in high risk groups in adult day care and community centres, specialty and nonspecialty out-patient clinics for adults and children, and hospital emergency departments.

Recommendation 21: Sufficient financial resources should be allocated to public health organizations and physicians for all aspects of vaccine delivery. When necessary, public health managers should prioritize nursing tasks on the basis of evidence-based practice to reallocate work for the accomplishment of immunization initiatives.

Strategies for promoting immunization programs

Recommendation 22: LCDC should act as a clearing-house for information on the implementation of pneumococcal immunization programs and program evaluation, and should provide each province and territory with a critique of current literature on the cost effectiveness of pneumococcal vaccination to assist decision makers in their determinations about the initiation of vaccination programs.

Recommendation 23: Information about adult immunization, including pneumococcal immunization, should be included in postgraduate training programs (Royal College of Physicians and Surgeons of Canada, Canadian College of Family Physicians), and in medical and nursing school curricula.

Recommendation 24: Information about pneumococcal immunization, pneumococcal disease, antibiotic resistance, and immunization record-keeping should be incorporated into continuing medical education activities such as conferences, lectures and grand rounds.

Recommendation 25: Adult immunization guidelines should be incorporated into practice guidelines at the provincial or territorial and federal levels.

Recommendation 26: Physicians should receive information (on a provincial or territorial basis) about Vaccine Associated Adverse Event (VAAE) surveillance system reports and be supplied with reporting forms. Timely feedback about adverse events should be provided to health care providers from both the national and provincial or territorial levels.

Recommendation 27: Periodic feedback should be given to the public about vaccine coverage, the impact of immunization programs on pneumococcal disease and adverse events associated with immunization.

Recommendation 28: Partnerships should be formed to promote pneumococcal immunization at the federal, provincial or territorial, and local levels with both professional organizations (eg, Canadian Medical Association, Canadian Paediatric Society, Canadian College of Family Physicians, Canadian Nurses Association, Canadian Association of Paediatric Hospitals, Canadian Infectious Disease Society) and nongovernmental organizations (eg, Canadian Public Health Association, Ro- 
tary Club, The Lung Association, Canadian Diabetes Association, Heart and Stroke Foundation, Canadian Cancer Society).

Recommendation 29: Promotional strategies should be developed at the local level using social marketing approaches, in order to reach different social groups (eg, targeting banks, churches, bingo halls, bus stops, pharmacies, post offices, etc) Recommendation 30: A competitive national market place for vaccine purchase should be promoted.

\section{Program evaluation and monitoring}

Evaluation of pneumococcal immunization programs will be critical to ensure that the objectives of the program have been met, including vaccine coverage, impact on disease burden, occurrence of adverse events, effectiveness of program delivery strategies, and awareness of and acceptance by health care providers and the public. The availability of measurable objectives for the control of pneumococcal disease and pneumococcal immunization programs will facilitate evaluation. Given the difficulties in determining rates of pneumococcal disease where $S$ pneumonaie is not isolated from a normally sterile body fluid, it will be useful to limit the scope to documented invasive disease; however, it is anticipated that immunization programs will prevent more than just these documented invasive infections.

\section{Strategies to evaluate immunization programs}

Recommendation 31: The effectiveness of program delivery strategies should be evaluated at all levels (national, provincial or territorial and local) using recognized methodologies.

Recommendation 32: Because both private and public delivery systems exist across Canada, each province and territory should evaluate how well national coverage goals are being achieved and modify its delivery system when needed.

Recommendation 33: Crude estimates of vaccine coverage should be obtained using common methodology across Canada (ie, doses distributed minus doses wasted as the numerator; adjusted census data for those aged 65 years and over, and high risk groups aged two to 64 years as the denominator). In addition, LCDC, in conjunction with the provinces and territories, should conduct periodic vaccine coverage surveys, including vaccine coverage in long term care facilities. A common methodology, including common definitions for numerator and denominator data, should be used for calculating vaccine coverage levels. Methods of improving vaccine coverage should be suggested.

Recommendation 34: Mortality data should be collected as a crude measure of disease impact.

Recommendation 35: The national VAAE surveillance system should continue to collect information not meeting full LCDC reporting criteria for severity ("other severe or unusual events" section). The provinces and territories should maintain the review of the reports as a centralized function.

Recommendation 36: Health care providers' acceptance of pneumococcal immunization programs should be assessed through knowledge, attitudes and practice surveys.

Recommendation 37: The general population should be surveyed to assess the effectiveness of promotional materials about pneumococcal immunization programs.
ACKNOWLEDGEMENTS: Health Canada thanks the planning committee, chairperson, speakers, chairs, rapporteurs, participants and Laboratory Centre for Disease Control (LCDC) staff, and contractors for their valuable contribution to this consensus conference. The LCDC extends sincere appreciation to Merck Frosst Canada Inc and Pasteur Mérieux Connaught Canada for supporting this consensus conference.

\section{REFERENCES}

1. Fedson DS, Musher DM. Pneumococcal vaccine. In: Plotkin SA, Mortimer EA Jr, eds. Vaccines, 2nd edn. Philadelphia: WB Saunders Co, 1994:517-64.

2. DiFabio JL, Homma A, DeQuadros C. Pan American Health Organization Epidemiologic Surveillance Network for Streptococcus pneumoniae. Microb Drug Resis 1997;3:131-3.

3. Reichler MR, Allphin AA, Breiman RF, et al. The spread of multiple-resistant Streptococcus pneumoniae at a day care centre in Ohio. J Infect Dis 1992;166:1346-53.

4. McGeer A, Green K, Landry L, et al. Assessing the potential impact of vaccination programs on invasive pneumococcal disease: Data from population-based surveillance. Can J Infect Dis 1999;10(Suppl A):24A-26A.

5. Kertesz DA, Senzilet L, Alagaratnum M, et al. Invasive pneumococcal disease in Canada 1996: Results from the Sentine Health Unit Surveillance system. Can I Infect Dis 1999;10(Suppl A):22A-23A.

6. Hansman D, Bullen MM. A resistant pneumococcus. Lancet 1967;ii:264-5. (Lett)

7. Appelbaum PC. Epidemiology and in vitro susceptibility of drug-resistant Streptococcus pneumoniae: an overview. Pediatr Infect Dis J 1996;15:932-9.

8. Lovgren M, Spika JS, Talbot JA. Invasive Streptococcus pneumoniae infections: serotype distribution and antimicrobial resistance in Canada, 1992-1995. Can Med Assoc J 1998; 158:327-31.

9. Lovgren M, Talbot JA. Antimicrobial resistant $S$ pneumoniae. Can J Infect Dis 1999;10(Suppl A):27A-29A.

10. Health Canada. Canadian Immunization Guide, 5 th edn. Ottawa: Minister of Public Works and Government Services Canada, 1998:140-3.

11. Prevalence of penicillin-resistant Streptococcus pneumoniaeConnecticut, 1992-1993. Morb Mortal Wkly Rep 1994;43:216-23.

12. Duchin JS, Breiman RF, Diamond A, et al. High prevalence of multi-drug resistant Streptococcus pneumoniae among children in a rural Kentucky community. Pediatr Infect Dis J 1995; 14:745-50.

13. Prevention of pneumococcal disease: Recommendations of the Advisory Committee on Immunization Practices (ACIP). Morb Mortal Wkly Rep 1997;46:1-23.

14. Nielsen SV, Henrichsen J. Incidence of invasive pneumococcal disease and distribution of capsular types of pneumococci in Denmark, 1989-94. Epidemiol Infect 1996;117:411-6.

15. Fedson DS, Shapiro ED, LaForce FM, et al. Pneumococcal vaccine after 15 years of use: another view. Arch Intern Med 1994;154:2531-5.

16. Shapiro ED, Berg AT, Austrian R, et al. The protective efficacy of polyvalent pneumococcal polysaccharide vaccine. N Engl J Med 1991;325:1453-60.

17. Fine MJ, Smith MA, Carson CA, et al. Efficacy of pneumococcal vaccination in adults: a meta-analysis of randomized controlled trials. Arch Intern Med 1994;154:2666-77.

18. Tengs TO, Adams ME, Pliskin JS, et al. Five hundred life-saving interventions and their cost effectiveness. Risk Anal 1995:15:369-90.

19. Sisk JE, Moskowitz AJ, Whang W, et al. Cost-effectiveness of vaccination against pneumococcal bacteremia among elderly people. JAMA 1997;278:1333-9.

20. DeWals P, Guay M, Drapeau J, et al. Pneumococcal immunization program: Cost-utility analysis for Quebec. Can J Infect Dis 1999; 10(Suppl A):46A-47A.

21. Baltussen RMPM, Ament AJHA, Leidl RM, et al. Cost-effectiveness of vaccination against pneumococcal pneumonia in The Netherlands. Eur J Public Health 1997; 7:153-61. 


\section{APPENDIX 1: GLOSSARY}

Cost effectiveness study: This form of economic analysis seeks to determine the costs and effectiveness of an activity or to compare similar alternative activities to determine the relative degree to which they will obtain the desired objectives or outcomes. The preferred action or alternative is one that requires the least cost to produce a given level of effectiveness or provides the greatest effectiveness for a given level of cost. In the health care field, outcomes are measured in terms of health status (24).

Cost-benefit study: An economic analysis in which the costs of medical care and the benefits of reduced loss of net earnings due to preventing premature death or disability are considered. The general rule for allocation of funds in a cost benefit analysis is that the ratio of marginal benefit (the benefit of preventing an additional case) to marginal cost (the cost of preventing an additional case) should be equal to or greater than one (24).

Cost-saving: Describing an intervention that is found to reduce medical expenses and improve health in an economic analysis.

Cost-utility: Describing a form of economic evaluation in which the outcomes of alternative procedures or programs are expressed in terms of a single 'utility-based' unit of measurement. A widely used measurement is the quality adjusted life year (24).

Effectiveness: A measure of the extent to which a specific intervention, procedure, regimen or service, when deployed in the field in routine circumstances, does what it is intended to do for a specified population (24).

Efficacy: The extent to which a specific intervention, procedure, regimen or service produces a beneficial result under ideal conditions. Ideally, the determination of efficacy is based on the results of a randomized controlled trial (24).
High risk groups for pneumococcal disease as defined by the National Advisory Committee on Immunization: High risk groups for pneumococcal disease include persons 65 years of age and older, persons over two years of age with asplenia, splenic dysfunction or sickle-cell disease, and persons over two years of age with the following conditions: chronic cardiorespiratory disease (except asthma), cirrhosis, alcoholism, chronic renal disease, nephrotic syndrome, diabetes mellitus, chronic cerebrospinal fluid leak, and human immunodeficiency virus infection and other conditions associated with immunosuppression (Hodgkin's disease, lymphoma, multiple myeloma, induced immunosuppression for organ transplantation) (10). Invasive pneumococcal disease: Pneumococcal infections identified by isolation of Streptococcus pneumoniae from normally sterile body fluid (ie, blood, and cerebrospinal, pleural, peritoneal or articular fluid).

Quality adjusted life year: An adjustment of life expectancy that reduces the overall life expectancy by amounts that reflect the existence of chronic conditions causing impairments, disability, and/or handicap as assessed from health survey data, hospital discharge data, etc. In practice, numerical weights representing severity of residual disability are established by the judgement of patients and health professionals (24).

Reduced susceptibility to penicillin: $S$ pneumoniae isolates identified using National Committee for Clinical Laboratory Standards (NCCLS) methods and breakpoints as having either high level resistance (minimum inhibitory concentration [MIC] $2 \mu \mathrm{g} / \mathrm{mL}$ or greater) or intermediate susceptibility (MIC 0.1 to $1.0 \mu \mathrm{g} / \mathrm{mL}$ ) to penicillin, or isolates identified using oxacillin screening as being nonsusceptible to penicillin (ie, zone size $19 \mathrm{~mm}$ or less) (25).

\section{APPENDIX 2: PARTICIPANTS OF PREVENTING PNEUMOCOCCAL DISEASE: A CANADIAN CONSENSUS CONFERENCE, FEBRUARY 16 to 18,1998}

Nicole Armstrong, Health Canada, Bureau of Infectious Diseases, LCDC, Ottawa, Ontario; Dr Adwoa Bentsi-Enchill, Research Section, Division of Immunization, Bureau of Infectious Diseases, LCDC, Ottawa, Ontario; Denise Bérubé, Merck Frosst Vaccine Division, Québec, Québec; Ivan Brophy, Department of Health \& Community Services of New Brunswick, Fredericton, New Brunswick; Dr Jerry Calver, Bureau of Biologics, LCDC, Ottawa, Ontario; Dr Maureen Carew, Medical Services Branch, Health Canada, Ottawa, Ontario, Dr Elizabeth Castañeda, Instituto Nacional de Salud, Santa Fe de Bogota, Columbia; Paresh Chaudhry, Merck Frosst Vaccine Division, Unionville, Ontario; Dr Michael Coulthart, Genetics, Bureau of Microbiology, LCDC, Ottawa, Ontario; Dr Patricia Daly, Vancouver/Richmond Health Board and Health and Epidemiology, University of British Columbia, Vancouver, British Columbia; Dr Shelley Deeks, Disease Control Service, Ontario Ministry of Health/LCDC, North York, Ontario; Gilles Delisle, Wyeth-Ayerst Canada, St-Laurent, Québec; Dr Philippe DeWals, Department of Maternal and Child Health, University of North Carolina, Chapel Hill, North Carolina; Dr Monique Douville-Fradet, Ministère de la santé et des services sociaux, Québec, Québec; Dr David Fedson, Pasteur Mérieux MSD, Lyon, France; Dr Giulio Ferrante, Vaccines Division, Bureau of Biologics, LCDC, Ottawa, Ontario; Dr Jacques Gaillat, Service de médecine interne et maladies infectieuses, Centre hospitalier de la région Annecienne, Annecy, France; Dr Ronald Gold, Pasteur Mérieux Connaught, North York, Ontario; Dr Karen Grimsrud, Alberta Health, Edmonton, Alberta; Dr Maryse Guay, Direction de la santé publique, Montérégie Health Unit, St Hubert, Québec; Dr Digby Horne, CDC Unit, Public Health Branch, Manitoba Health, Winnipeg, Manitoba; Dr Greg Horsman, Provincial Laboratory, Saskatchewan Health, Regina, Saskatchewan; Dr Louise P Jetté, Laboratoire de santé publique du
Québec, Ste-Anne-de-Bellevue, Québec; Dr Barbara H Kawa, Region of Peel and Canadian Public Health Association, Brampton, Ontario; Dr Daniel Kertesz, Bureau of Infectious Diseases, LCDC, Ottawa, Ontario; Dr Arlene King, Communicable Disease Epidemiology, British Columbia, Ministry of Health, Vancouver, British Columbia; Joan Lindsay, Aging-related Diseases Section, Division of Aging and Seniors, Health Canada, LCDC, Ottawa, Ontario; Kay Maclsaac, Public Health Services, Province of Nova Scotia, Bedford, Nova Scotia; Peggy MacLeod, College of Nursing, Health Sciences Building and Canadian Nurses Association, Saskatoon, Saskatchewan; Pat Mandl, Department of Health \& Social Services, Communicable Disease Control Unit, Health \& Social Services Yukon, Whitehorse, Yukon; Dr Victor Marchessault, National Advisory Committee on Immunization, Ottawa, Ontario; Dr Thomas Marrie, Queen Elizabeth II Health Sciences Centre, Halifax, Nova Scotia; Dr Michael McDonald, Infectious Diseases Working Group, The Lung Association, Halifax, Nova Scotia; Dr Allison McGeer, Mount Sinai Hospital and Canadian Infectious Disease Society, Toronto, Ontario; Dr John Millar, British Columbia Ministry of Health, Victoria, British Columbia; Julie Morrison, Community Health, Aboriginal Nurses Association of Canada, Inuvik Regional Hospital, Inuvik, Northwest Territories; Dr Monika Naus, Disease Control Service, Ontario Ministry of Health, North York, Ontario; Dr Liana Nolan, Saskatchewan Health, Saskatoon, Saskatchewan; Ms Cathy O'Keefe, Disease Control \& Epidemiology, Department of Health, Newfoundland, St John's, Newfoundland; Dr Peter Paradiso, Wyeth-Ayerst Lederle, Vaccine and Paediatric, West Henrietta, New York; Ms Karen Pielak, British Columbia Centre for Disease Control and Canadian Nursing Coalition for Immunization, Vancouver, British Columbia; Dr Maurice Poulin, Ministère de la santé et des services sociaux, Québec, Québec; Dr Kirsten Rottensten, 
Ontario Ministry of Health, Toronto, Ontario; Marilyn Sark, Aboriginal Nurses Association of Canada, Lennox Island, Prince Edward Island; Dr David Scheifele, Pediatric Infectious Diseases, Vaccine Evaluation Center and Canadian Paediatric Society/IMPACT, Vancouver, British Columbia; Dr Walter Schlech, National Advisory Committee on Immunization, Halifax, Nova Scotia; Dr Jeff Scott, Nova Scotia Department of Health, Halifax, Nova Scotia; Yvette Sentenne, National Advisory Council on Aging, Montreal, Québec; Dr Jane Sisk, Division of Health Policy and Management, Columbia University School of Public Health, New York, New York; Dr John Spika, Bureau of Infectious Diseases, LCDC, Ottawa, Ontario; Dr Lamont Sweet, Department of Health \& Social Services, Prince Edward Island Department of Health \& Social Services, Charlottetown, Prince Edward Island; Dr Jim Talbot, Provincial Laboratory of Public Health of
Northern Alberta, University of Alberta Hospital, Technical Advisory Committee, Edmonton, Alberta; Dr Susan Tamblyn, Medical officer of Health, Perth District Health Unit, Stratford, Ontario; Brenda Thomas, Assembly of First Nations, Ottawa, Ontario; Dr Roger Thomas, Department of Family Medicine, University of Ottawa and College of Family Physicians of Canada, Ottawa, Ontario; Dr Mary Vearncombe, Canadian Association of Medical Microbiologists and Department of Clinical Laboratories, Women's College Hospital, Toronto, Ontario; Bruce R Wallace, Health \& Social Services, Prince Edward Island, Charlottetown, Prince Edward Island; Dr John Waters, Alberta Health, Edmonton, Alberta; Ms Wanda White, Department of Health \& Social Services, Northwest Territories, Yellowknife, Northwest Territories; Dr Cindy Whitney, Centers for Disease Control and Prevention, Atlanta, Georgia
22. Health Canada. Canadian Consensus Conference of a National Immunization Records System. Can Commun Dis Rep 1998;24:137-40.

23. Guay M, DeWals P, Hébert R. Pneumococcal immunization program in Montérégie Québec: feasibility study. Can J Infect Dis 1999;10(Suppl A):53A-56A.
24. International Epidemiological Association, Inc. In: Last JM, ed. A Dictionary of Epidemiology, 3rd edn. New York: Oxford University Press, Inc, 1995.

25. Defining the public health impact of drug-resistant Streptococcus pneumoniae: report of a working group. Morb Mortal Wkly Rep 1996;45:1-20.

This article was published simultaneously by the Canadian Communicable Disease Report 1999;25-4:25-36. 


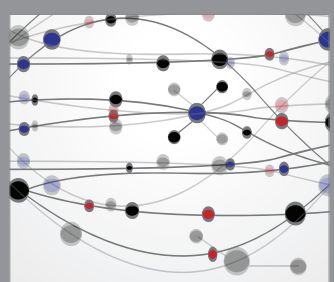

The Scientific World Journal
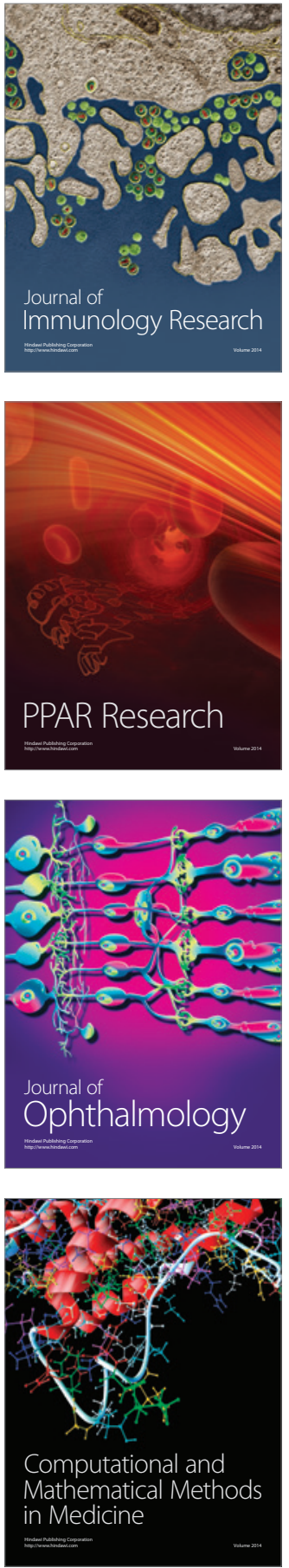

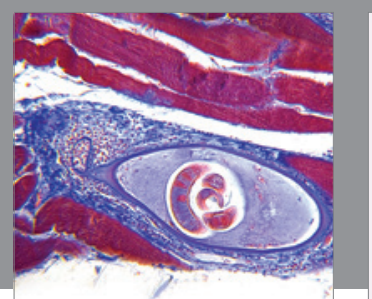

Gastroenterology Research and Practice

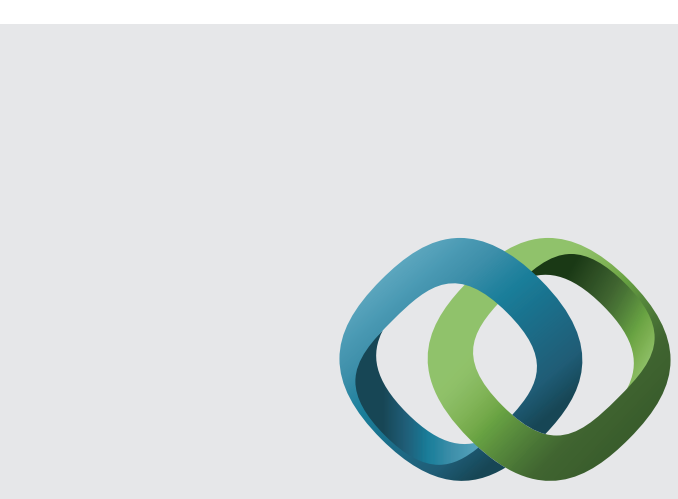

\section{Hindawi}

Submit your manuscripts at

http://www.hindawi.com
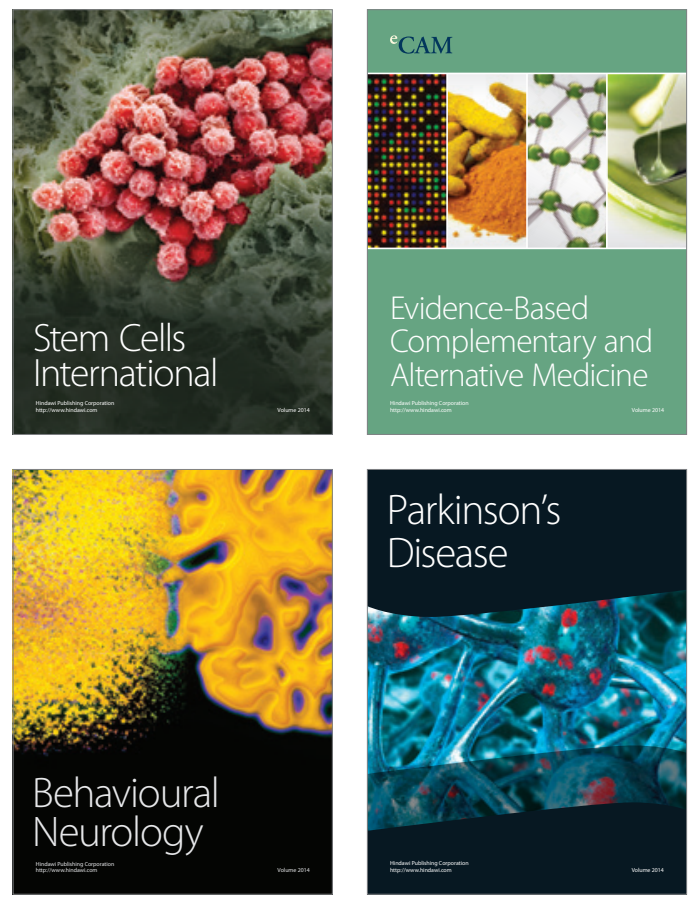
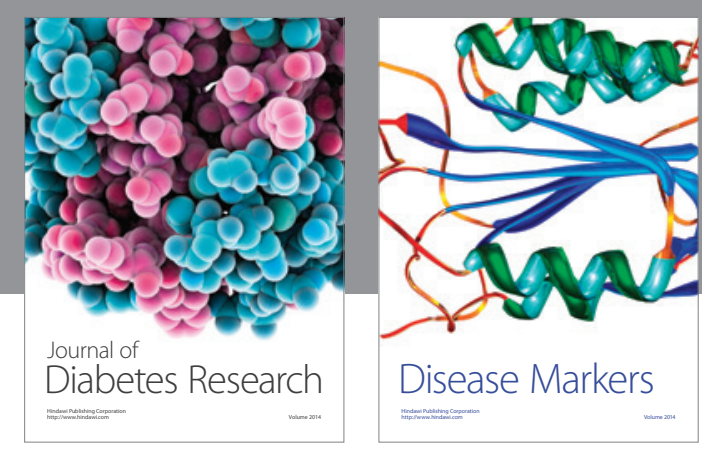

Disease Markers
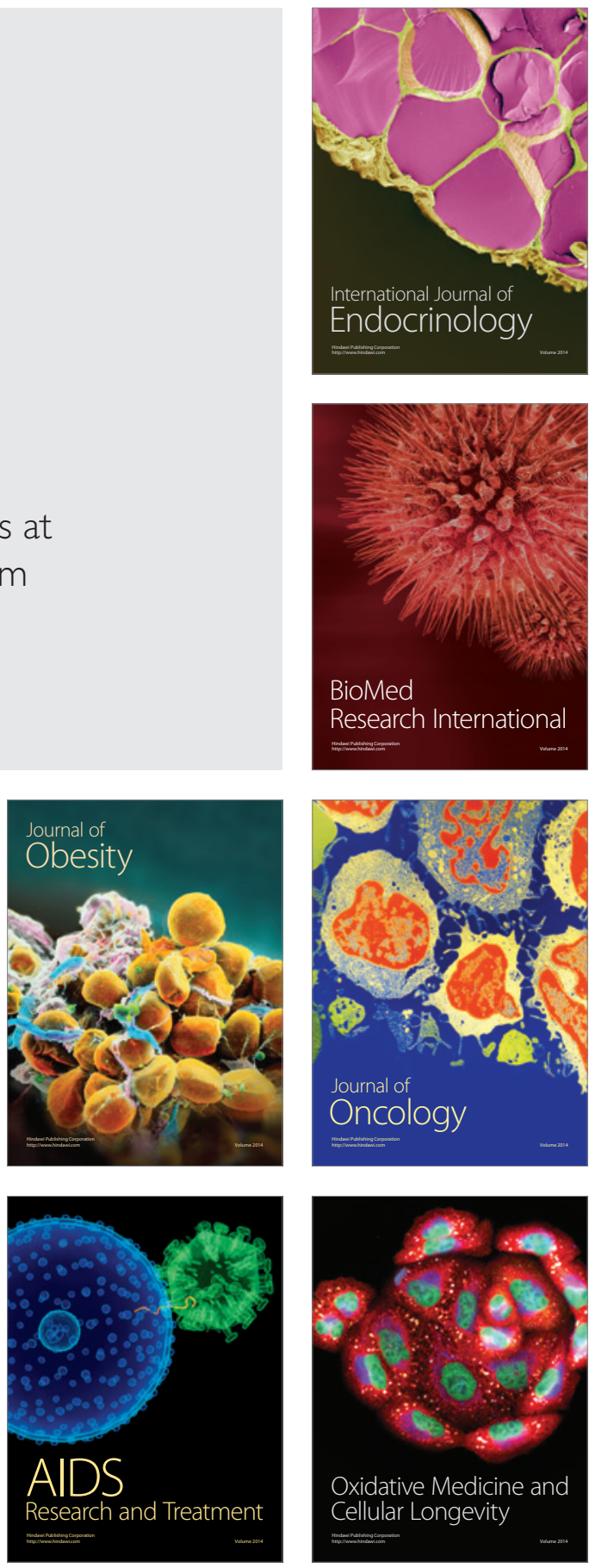